\title{
La lectura y la escritura en los procesos de aprendizaje de los estudiantes de educación superior $^{1}$
}

\author{
Reading and writing in the learning \\ processes of students in higher education
}

\author{
Patricia Ramírez Otálvaro ${ }^{2}$ \\ Semillero Enseñanza Aprendizaje en Educación Superior —EAES— \\ E-mail: semilleroensenanzaaprendizaje@gmail.com
}

\section{Resumen}

Los integrantes del semillero de investigación Enseñanza Aprendizaje en Educación Superior ${ }^{3}$, como parte del ejercicio investigativo, han realizado un rastreo documental sobre las prácticas de lectura y escritura, y su incidencia en el aprendizaje y la construcción de conocimiento disciplinar; encontrando tendencias conceptuales y, procedimientos problemáticos o situaciones heredadas desde la escuela y a las cuales la educación superior, no les está dando solución; material compilado con el propósito de motivar la búsqueda de prácticas sistemáticas y responsabilidades compartidas entre los encargados de la enseñanza en educación superior, referidas con la promoción y la sostenibilidad de una cultura académica, que promueva la lectura y la escritura, como uno de los ejes transversales a la apropiación y reconstrucción de saberes.

Palabras clave: Educación superior, Enseñanza, Lectura, Escritura, Aprendizaje, Conocimiento

Texto logrado con el apoyo de los estudiantes de Licenciatura en Educacion Básica con énfasis en Humanidades y Lengua Castellana (LEBHLC): Stephanie Pérez Ortiz, y Santiago Alzate Hincapié, en las fases de planeación y elaboración de borrador o pre-escritura.

2 Docente Asociada del Tecnológico de Antioquia, Institución Universitaria. Licenciada en Educación Especial, Magíster en Educación, Doctora en Psicología de la Educación, Cultura y Sistemas Semióticos.

3 Semillero de Investigación EAES, conformado por grupo de estudiantes (LEBHLC) responsables de la búsqueda, selección, discusión, análisis de los tópicos teóricos y problemáticos que han dado lugar a esta composición: Sulay Jansey Lopera, Yaneth Orrego Alarca, Deisy Luján Sánchez, Julieth Natalia Úsuga, Lina Marcela Acosta, Sebastián Zánder Londoño, Stephanie Pérez Ortiz, y Santiago Alzate Hincapié. E-mail: semilleroensenanzaaprendizaje@gmail.com 


\section{Patricia Ramírez. Otálvaro}

\section{Abstract}

The members of the seedbed of investigation Education Learning in Top Education, as part of the exercise investigativo, have realized a documentary tracking on the practices of reading and writing, and his incident in the learning and the construction of knowledge to discipline; finding conceptual trends and, problematic procedures or situations inherited from the school and to which the top education is not giving solution; compiled with the intention of motivating the search of systematic practices and responsibilities shared between the managers of the education in top education, recounted with the promotion and the sustainability of an academic culture, which promotes the reading and the writing, as one of the transverse axes to the appropriation.

Keywords: Higher Education, Teaching, Reading, Writing, Learning and Knowledge

\section{Aproximaciones conceptuales}

La alfabetización, en si misma y por si misma, rara vez conduce a cambios significativos en el estilo cognitivo y el aprendizaje. Lo esencial son las prácticas $y$ usos en los que interviene esta alfabetización [...]. No basta con que los estudiantes estén alfabetizados formalmente; también es necesario que utilicen la alfabetización de una manera que sea pertinente para el objetivo de la educación formal.

(Kozulin, 2000, p. 146).

Se concibe la lectura como la puerta al conocimiento, la herramienta privilegiada de la inteligencia, el instrumento para identificar, evaluar y contrastar el propio pensamiento o cuestionar, reforzar y modificar lo aprendido. Es un proceso a partir del cual se logra desentrañar la estructura simbólica y argumental del texto y los posibles universos que en él se contemplan de manera explícita e implícita, lo cual demanda el procesamiento secuencial de descifrar, inferir, re-construir y de-codificar, la puesta en escena de operaciones de pensamiento tales como representar, verificar, inferir, y construir hipótesis, la relación entre procesos mentales lingüísticos como significado, sintaxis, y vocabulario, y los procesos extralingüísticos relacionados con situación e intención comunicativa y modo de tratar la información (De Zubiría, 1997, 1998; Solé, 2012).

La lectura comprensiva requiere entonces que el lector procese individualmente los contenidos de las cláusulas y las frases (nivel ejecutivo y funcional), integre la información de estas en unidades más globales de significado (nivel instrumental), y elabore la coherencia con base en las proposiciones expresadas entre lo leído y el conocimiento que está almacenado en su memoria (nivel epistémico o lectura crítica). Es decir, el fin de leer no es solo comprender la estructura semántica y las representaciones significativas subyacentes al texto, sino además grabar en la memoria a largo plazo los conocimientos adquiridos durante la lectura o re-estructurarlos según las estructuras de pensamiento y el conocimiento previo (De Zubiría, 1997, 1998; Martínez Fernández, 2004; Van Dijk, 1985).

Postulan los autores que para el caso de estudiantes de educación superior, la lectura 
debería constituirse en un proceso para fortalecer y potenciar lo aprendido a partir de la extracción o la elaboración de macro-proposiciones y sus relaciones, modelos gráficos, estructuras semánticas, reconstrucción de textos escritos y orales, cambio de sentido global o invención de las composiciones con base en reglas determinadas o esquemas de múltiples relaciones, siguiendo el estilo de las producciones filosóficas, científicas, literarias o tecnológicas, con sus tesis y enlaces ocultos o implícitos; las características del lector son tan importantes para la lectura como las características del texto.

Por tanto, "saber leer no presupone necesariamente saber leer de forma crítica, para pensar o generar conocimiento; ese potencial solo se concreta cuando el lector participa en situaciones que le exigen ir más allá del texto dado y adentrarse en su análisis, contraste y crítica” (Solé, 2012, p. 54). Dicha tarea alcanza un nivel de abstracción tan elevado que requiere ser enseñado de acuerdo con las operaciones intelectuales propias del pensamiento formal (sintetizar, analizar, relacionar, deducir, inducir, formular hipótesis, etc.), y con la exigencia de los textos, los cuales deben ser leídos buscando desbordar y superar su significado (textual), para acceder a su sentido (con-textual o meta-textual).

Se ratifica entonces que

[...] leer es la llave de la puerta al conocimiento. Aun con el actual auge de las computadoras, aun cuando digan que la multimedia reemplazará más temprano que tarde a los libros, siempre habrá necesidad de leer. Es cierto: tal vez en el futuro, leer no signifique decodificar los mismos signos que hoy conocemos, pero siempre existirá la lectura, como siempre existirá el pensamiento. Tal vez la inteligencia humana encuentre uno o varios canales más adecuados que el lenguaje verbal para expresarse, pero nunca dejará de producir ideas, conocimiento (De Zubiría, 1997, p. 3).

De otra parte, la escritura se concibe como creación científico-cultural: posibilidad de crear y transferir al papel o a cualquier otro medio la representación de universos simbólicos, ideas generales, abstractas y globales, mundos posibles que demuestran los procesos de pensamiento, la inteligencia humana, la construcción y la re-construcción del conocimiento; como proceso y producto que ha llevado a los sujetos y a las sociedades a diversas transformaciones; como sistema comunicativo e informativo por excelencia de los pueblos, y esencia constitutiva de la humanidad y las identidades sociales (Cardona, 1994; De Zubiría, 1998).

Es la habilidad que permite al sujeto convertir los códigos tipográficos y los grafemas en letras, palabras, oraciones, proposiciones y unidades agrupadas hasta lograr un texto global con estructura semántica, significativa y conceptual que refleja el sentido general del discurso; instrumento que permite al autor expresar pensamientos, inquietudes o cualquier información para sí mismo o el receptor (autocomplacencia personal, ansiedad, imaginación y curiosidad), independiente de la orientación final de la producción escrita describir, exponer, narrar, explicar o argumentar-. Por tanto, no todos los textos tienen el mismo fin, intención, método, formato y mecanismos de comunicación y elaboración; algunos demandan información, explicación detallada, descripción específica o demostración, y otros, implican el impulso, la imaginación y la fantasía (Sánchez, 2007; De Zubiría, 1997, 1998; Valery, 2000). 
De aquí que la escritura sea el uso consciente, reflexivo y controlado del código escrito para generar eventos comunicativos, interpretar, construir mundos existentes o imaginar escenarios posibles. El seguimiento a la producción escrita de los expertos señala la toma de conciencia sobre sus composiciones, diferenciada por momentos ${ }^{4}$ que se planifican, interrelacionan, superponen, reiteran e interactúan entre sí, con los conocimientos sobre el mundo y sobre el lenguaje de quien escribe (Sánchez, 2007; Flower y Hayes, 1984).

"La escritura permite convertir todo el misterio de la vida, en arte expresivo y artístico a través de la palabra" (Sánchez, 2007, p. 71). La palabra como célula del pensamiento es la responsable de generalizar las cosas, de incluirlas en determinadas categorías y demostrar su compleja función intelectual generalizadora, lo que la convierte en el repertorio básico de la escritura, constructora de los significados, la actualización y la elección del registro, la situación de comunicación, el estilo $^{5}$ y el género textual ${ }^{6}$. Aunque parezca reiterado, el significado de cada palabra cambia no solo la estructura semántica en el proceso de lectura o escritura, sino el sistema psíquico que está detrás de ella (operaciones de pensamiento), y demanda al sujeto la búsqueda de significados a y entre los conceptos para almacenarlos y recuperarlos, en coherencia con cada contexto comunicativo (Valery, 2000).

Desde tal perspectiva, las formas de expresión escrita son tan variadas como las formas de expresión oral, aunque la producción escrita es mucho más exigente social y culturalmente en el manejo del repertorio léxico, la propiedad gramatical y la corrección ortográfica, y exige del escritor perfección normativa, formación conceptual, manejo de la lengua, memoria semántica, abstracción mental, conocimiento cultural, técnicas de redacción, revisión, deseo, habilidad, esfuerzo, disciplina y práctica constante; tales insumos le permiten perfeccionarse en la composición, redacción y elección de palabras para combinarlas de manera coherente, y así lograr expresar, recrear, comunicar y transformar el sentido y el significado o asumir método y estilo discursivos.

No todos los buenos lectores son buenos escritores; para ser un buen escritor hay que leer como escritor, es decir, no limitarse a comprender y descubrir qué hace interesante, oportuno, coherente y correcto eso que se lee. Asumir la posición de un escritor potencial al leer, permitiría adquirir progresivamente las habilidades que exige la escritura. Desde esta perspectiva, la escritura comprende la lectura y constituye por tanto una tarea más compleja: leer para escribir y, luego, escribir a partir de lo que se adquirió leyendo (Aterrosi, 2010, p. 192).

Las tendencias teóricas enunciadas de manera genérica develan que el sujeto, al interior de su grupo social, se apropia de herramientas como la lectura y la escritura que le facilitan la adaptación, la creación y la modificación de la realidad. Son procesos distintos que se correlacionan e inciden en el procesamiento y la comprensión de la información, la construcción de conocimientos y la autorregulación del proceso de aprendizaje, porque se convierten en

\footnotetext{
Momentos que siguen los expertos al escribir: pre-escritura, planificación, puesta en texto, revisión, corrección y edición. Modelo de la escritura como proceso cognitivo (Flower y Hayes, 1984).

5 Estilo: florido, retórico, conciso, amplificado, didáctico.os.

6 Género: narrativo, descriptivo, expositivo, didáctico, instructivo y argumentativo.
} 
instrumentos para aumentar, revisar, sostener, sustentar, afianzar, mantener, transformar y usar el propio saber, y constituyen competencias básicas que garantizan la capacidad para seguir aprendiendo durante toda la vida. "El verdadero sentido y significado social del acto de escribir y leer es transferir estructuras semánticas o sistemas de conocimiento entre los individuos" (De Zubiría, 1998, p. 195).

De manera complementaria,

[...] se entiende el proceso de aprendizaje como una serie compleja de actividades sociales e individuales, mediante las cuales el estudiante se apropia de la cultura de su entorno para enfrentar de manera consciente y creativa, problemas de los ámbitos en los que debe desenvolverse [...] ocurre cuando se logra relacionar de manera coherente, la nueva información con los conocimientos y las experiencias anteriores, esto, unido a la disposición de aprender (Pozo, Scheuer y Mateos, 2009, p. 28).

En consecuencia, el aprendizaje siempre está mediatizado por procesos interactivos complejos en los que el sujeto interpreta a partir de su estructura cognoscitiva (conceptual, metodológica, estética, actitudinal y axiológica), y transforma significados y formas de significar de acuerdo con su entorno socio-histórico-cultural. Se confirma que todo aprendizaje implica una doble dimensión de interdependencia: la construcción de significados y la atribución de sentido a los contenidos aprendidos. Además, cuando los estudiantes involucran en el aprendizaje el afecto, la emoción, el interés, el sentido de competencia y la autonomía, no solo modifican la comprensión de las cosas, sino que dicho aprendizaje comienza a formar parte de la realidad, y transforma el sentido sobre la misma (Martínez y Galán, 2000; Solé y Coll, 2006; Ugartetxea, 2001).

Se reconocen así las correlaciones entre el aprendizaje y las prácticas de lectura (profunda), porque son estas, las que habilitan al estudiante para relacionar textos, teorías, conceptos, habilitando el análisis, la interpretación, la categorización, la transferencia, reconstrucción y retroalimentación teoría-práctica - prácticateoría, y la producción de textos verbales o escritos. De manera alterna, los procesos exigidos, tanto para demostrar el aprendizaje como para escribir, exigen recuperar los conceptos almacenados en la memoria, relacionarlos, compararlos o cuestionarlos con el propósito de reformularlos y usarlos como instrumentos mentales, desde los cuales interpretar objetos de estudio y, comunicar el dominio compresivo de los mismos, en función del propósito del texto, la tarea de aprendizaje, el escenario y el auditorio.

\section{Escenarios problemáticos}

En una cultura donde leer ocupa uno de los últimos lugares en la lista de las cosas que hacen felices a los ciudadanos, hablar de lectura puede parecer un contrasentido.

(De Zubiría, 1997, p. 3).

La problemática gira en torno a las concepciones y prácticas de lectura y escritura de los estudiantes y los profesores de educación superior, como prácticas sociales y académicas ya adquiridas, y a la permanencia en el tiempo de culturas 
académicas que invisibilizan el cuándo, el quién y el cómo enseñar, evaluar o fortalecer los procedimientos relacionados con la comprensión de lectura, la producción escrita y su interacción con el aprendizaje disciplinar.

La concepción sobre la responsabilidad de enseñar la lectura y la escritura suele reducirse a los dos primeros ańos escolares, acercamiento fonético ${ }^{7}$ y silábico ${ }^{8}$ que no equivale a aprender a leer o escribir. ¿Cuántos profesores, durante los años de escolaridad posteriores, se comprometen con la enseñanza y el fortalecimiento de las habilidades para comprender e interpretar textos o producirlos a partir de criterios específicos? Los profesores suponen que los estudiantes ya poseen las técnicas y las habilidades necesarias para leer y escribir, y que no es su responsabilidad estimular, facilitar o beneficiar su posible desarrollo o potenciación.

En últimas, suelen utilizar tales prácticas más como instrumentos de registro para proveer insumos de evaluación (tareas, talleres u otras herramientas de evaluación), que como prácticas cognitivas, complejas y necesarias requeridas en la apropiación de conocimientos, la adquisición de competencias y el aprendizaje autónomo, práctica constante para el resto de la vida (Carlino, 2004, 2005a; Solé, 2012). Se olvida que "los modos de leer y escribir de los estudiantes son un efecto de las condiciones pedagógicas y didácticas que la universidad promueve, así como el tipo de demandas que plantea” (Rincón, Narváez y Pérez, 2011, p. 1).

La utilidad de las prácticas suele limitarse al cumplimiento de propósitos semejantes a evaluar el aprendizaje en formatos como resúmenes, esquemas, reseñas, reporte de textos o disertaciones orales, donde se priorizan aspectos ortográficos, puntuación o sintaxis oracional, sin revisar aspectos complejos tales como coherencia, cohesión, contextualización y estructura relacional; la tendencia señala que los profesores sancionan en lugar de retroalimentar, situación que limita el protagonismo del estudiante en su proceso de aprendizaje.

De allí se desprenden inquietudes referidas a ¿Cuántos profesores de educación superior se preguntan o sistematizan indicadores y factores incidentes en el desempeńo de los estudiantes, en lo relativo a producción escrita o comprensión de lectura y su correlación con el aprendizaje? ¿Quiénes sugieren formatos o modelos para que los estudiantes alcancen la comprensión de lectura y la escritura en coherencia con una tipología o estilo determinados? ¿Quiénes proponen prácticas de lectura y escritura sistemáticas, como ejes de transformación y uso del conocimiento o actúan como mediadores y modelos en el fortalecimiento de las competencias que subyacen a dichos procesos? Nadie puede enseñar lo que no sabe, por tanto, nadie podrá enseñar a leer ni a escribir si desconoce los mecanismos inherentes al acto lector o a la producción escrita.

Por su parte, los estudiantes acuden a la escritura para organizar la información de lo leído, y acumulan la experiencia de reconocer documentos y páginas de manera atomizada, sin elementos para identificar lo leído o lo escrito desde el diálogo entre conceptos, postulados, hipótesis o investigaciones; siendo así, los resultados no son los esperados, se hallan producciones desestructuradas, sin intención comunicativa, confusas entre una

7 Memoria semántica y comprensiva sobre la escritura ideográfica (representación gráfica de objetos y acciones); el lenguaje como un signo gráfico para cada sonido(gramática, semántica y pragmática), y la globalidad del universo simbólico para ingresar a la cultura (De Zubiría, 1997).

8 Encontrar el significado a las grafías al interior de los conceptos, almacenarlos y recuperarlos convertidos en palabras; donde el significado de cada palabra cambia no solamente su estructura semántica, sino el sistema psíquico que está detrás de la misma (instrumentos del conocimiento, operaciones del intelecto), aunque no todas las palabras implican un concepto determinado-colores (De Zubiría, 1997

Revista Senderos Pedagógicos N. $^{\circ} 3$ • Enero - Diciembre 2012 
idea y otra, sin calidad en el uso del léxico y las normas ortográficas, que develan falta de reconocimiento sobre la información, poca comprensión sobre los tópicos temáticos y una lectura superficial y descontextualizada (Ochoa y Aragón, 2004). Este planteamiento es observable en los resultados de las pruebas nacionales "Saber Pro", las cuales, desde el año 2004, señalan un bajo nivel de lectura crítica ${ }^{9}$ y escritura ${ }^{10}$, con especial preocupación para los estudiantes de los programas de educación -revisar sistematización de datos en la Web del ICFES: http://www.icfes. gov.co/investigacion/informes-de-resultados-deevaluaciones-nacionales/saber-pro-.

A propósito de otras causales, se plantea la falta de compresión de lectura, porque los estudiantes en la educación superior se ven obligados a enfrentarse a otro tipo de textos, mucho más cuando provienen de una cultura lectora o escritora diferente, cuyos objetivos, reglas y materiales son mecánicos y no exigen comprensión o utilización de información. Es decir, carecen de conocimientos previos y propósitos de lectura, no tienen modelos sobre la situación o lo que informa el texto, ni macroreglas para seleccionar los datos importantes de los superficiales, construir y transformar información o generar nuevas macro-estructuras (Carlino, 2004; Van Dijk, 1985).

En lo relativo a la escritura, los estudiantes de educación superior -escritores novatos-, están predispuestos frente a la producción escritural y no siempre cuentan con la motivación para escribir. Las composiciones parecen sumarios más que síntesis analíticas argumentativas, no tienen en cuenta la perspectiva del lector ni el objetivo de la escritura; no consideran las exigencias retóricas para producir un texto adecuado; suelen desaprovechar el potencial epistémico de la escritura; se han acostumbrado a revisar los textos solo en forma lineal, por partes localizadas y poco sustantivas, e incurren en la dilatación o postergación del momento para comenzar a escribir, esbozar ideas, sugerir perspectivas y posturas de comprensión, terminando por realizar composiciones sin estilo. A ello se suma la angustia de escribir, la inseguridad de cómo hacerlo o la representación de estar haciendo una tarea equivocada, vaga o sin sentido (Carlino, 2004).

Otros estudios señalan que los novatos se conforman con activar en su mente el tema que quieren escribir y de manera inmediata generan el texto, permeado de ideas instantáneas, sin la intención de relacionar contenido con retórica o transformar conocimiento en coherencia con el destinatario o el sentido de la composición; la producción escrita se publica sin haber estado mediada por recursos tales como planeación, revisión, re-escritura o corrección (Bereiter y Scardamalia, 1987, citados por Aterrosi, 2010).

Tal caracterización señala un estudiante que no dispone de las habilidades necesarias para acceder a las formas culturales superiores de las cuales depende el desarrollo científico, tecnológico y artístico de una nación; por tanto, le es difícil acceder a procesos de lectura meta-textual, que demanda ir más allá del significado evidente del texto para desentrañar motivaciones e ideologías del escritor, contrastar, contraponer o establecer relaciones socioculturales con otros sistemas de ideas o estilos de composición, según corresponda (De Zubiría, 1998). Al mismo tiempo, es un estudiante que desconoce las fases de la lectura y la escritura, por tanto no las aplica, no tiene hábitos adecuados para el nivel

\footnotetext{
9 “Desempeños asociados con la lectura, pensamiento crítico, lectura analítica y reflexiva, comprensión de los planteamientos expuestos en un texto e identificación de perspectivas y juicios de valor, identificación y recuperación de información presente en uno o varios textos, construcción de sentido global del texto, establecimiento de relaciones entre enunciados y evaluación de la intencionalidad del escrito" Competencias Genéricas, Saber Pro (MEN, 2009)

10 "Comunicación de ideas, teniendo en cuenta la elaboración de la intención y el propósito, la claridad de la estructura y la organización y el manejo del lenguaje y las convenciones". Competencias Genéricas, Saber Pro (MEN, 2009).
} 
de exigencia académica en el que se encuentra o no usa estrategias de aprendizaje cognitivas, ni meta-cognitivas que les facilite la comprensión, reflexión, autoevaluación y corrección de lo que lee y escribe, hallando como resultado la lectura superficial, la escritura sin calidad y el aprendizaje a corto plazo (Cano, 2005; Carlino, 2005a; Martínez y Galán, 2009; Ugartetxea, 2001). La pobreza en los discursos orales y escritos se justifica, por último, por la incidencia de las nuevas formas de comunicación social e histórica (chat, sms, correos, twitter, fecebook), el uso de extranjerismos, anglicismos, descuidos idiomáticos o incorrecciones, asuntos que de manera continua o a largo plazo pueden limitar la memoria lingüística, volviéndola efímera o superficial; mucho más cuando los estudiantes de educación superior no tienen una práctica sistemática que les garantice el dominio del vocabulario, las estructuras morfosintácticas de manera integrada con las referencias culturales formales (Sánchez, 2007).

Se exponen, para concluir los escenarios problemáticos, los diseños curriculares en las instituciones de educación superior de Iberoamérica. Carlino (2005a) señala que las técnicas de escritura y lectura se han asignado como responsabilidad única para los cursos del área del lenguaje (lengua materna, habilidades comunicativas, técnicas de escritura), y por tanto los alumnos escriben poco o lo hacen sin recibir la orientación por parte de los profesores, y de este modo desconocen la escritura y la lectura como procesos transversales facilitadores de la comunicación y el diálogo con los otros, con los diversos saberes, textos y contextos.

Valga señalar, antes de pasar a las posibilidades desde el hacer, que las prácticas de lectura y escritura se encuentran correlacionadas con las prácticas discursivas, no menos importantes como escenario conceptual y problemático por su ausencia de la enseñanza en la educación superior. El estudio de Gutiérrez (2012), demuestra la falta de reflexión sobre la oralidad o la práctica discursiva oral de los estudiantes como actividad del lenguaje, en su manifestación, configuración, funcionalidad y uso en los contextos académicos y socioculturales.

En últimas, se evidencian escenarios, concepciones y prácticas discursivas, de lectura y de escritura en los procesos de enseńanza aprendizaje de la educación superior, que dejan inquietudes a propósito de su incidencia en el aprendizaje, la reconstrucción de conocimiento y sus contextos de uso y comunicación cotidiana o disciplinar; es decir, las prácticas objeto de discusión, están avaladas en la enseñanza, como medios para evaluar el contenido de los textos (lo que se les pide que escriban, que lean o que expongan) y ocasionalmente la normatividad, más que como herramientas para disertar, apropiarse o representar o lo saben qué saben, lo qué saben hacer, lo qué piensan de lo que saben, y lo qué hacen con lo que saben.

\section{Posibilidades prácticas}

En una época en la que ya no se discute el derecho a que la alfabetización vaya más allá de la adquisición de letras y palabras, en la que se habla de "alfabetizaciones" en plural y de su aprendizaje a lo largo de toda la vida, es preciso conocer dónde estamos, cuánto y qué nos falta para alcanzar esas metas. (Valdés, 2010, p. 13).

En el "deber ser" y las posibilidades de usar la información encontrada, quedaría la invitación a los responsables de la gestión académica y administrativa de los programas en educación superior, los docentes y los propios estudiantes, para realizar una valoración sobre las concepciones y las prácticas de lectura y escritura, y los lugares 
que ocupan en el proceso de aprendizaje disciplinar; con miras a que tal análisis y sus resultados, redunden en el diseño de políticas académicas y estrategias de enseñanza, evaluación y aprendizaje, encaminadas al fortalecimiento de las competencias implícitas en la lectura y la escritura y, su transversalidad con el cambio conceptual o la apropiación de conocimiento académico, epistemológico, técnico, científico y sociocultural (Carlino, 2005b; Aterrosi, 2009; Zambrano, 2010); y en la gestión de proyectos para sistematizar, replicar o estimular experiencias innovadoras o didácticas específicas encaminadas a tal fin.

$\mathrm{Al}$ respecto, los diseños curriculares y didácticos de los diversos programas deberían reconocer las funciones del lenguaje en uso, y la lectura y la escritura como actividades sociales dependientes de las estructuras sociales letradas (tomando como referencia la escritura experta o la buena lectura), y como herramientas básicas que faciliten la transformación del conocimiento y su aplicación en la solución de problemas del contexto social, profesional y laboral.

Integrar además, modalidades de enseñanza y evaluación del lenguaje, orientadas hacia las operaciones de pensamiento, las estrategias de aprendizaje y las intenciones comunicativas de lo aprendido; modalidades mediadas por situaciones explicitas, donde el enseñante-experto provea al aprendiz-novato, de la estructuración de recursos discursivos y textuales en función de los diferentes modos de circulación (social, académica y cultural), destinatarios, sentidos y fines de la producción textual y académico; favoreciendo la reconstrucción de aprendizajes, y las competencias de lectura (pre-lectura, lectura, relectura), escritura (pre-escritura, revisión, corrección y mejora continua), autocrítica y autorregulación de aprendizajes.

Asimismo, los profesores de educación superior, en especial los responsables de la formación de maestros, han de aprovechar las investigaciones y los estudios referidos al sistema de escritura, la función de la lectura, las estrategias cognitivas y meta-cognitivas implicadas en la lectura y la escritura, la incidencia de ambas prácticas en el proceso de aprendizaje, entre otras; reconocimiento de teorías y procedimientos que podrían aportar en la modificación de procedimientos y concepciones sobre el cómo mejorarlas en forma permanente y transversal, e integrarlas al proceso de aprendizaje disciplinar y sus particularidades epistemológicas y discursivas (Carlino, 2005b).

En el mismo nivel se encontraría la responsabilidad del estudiante, quien se debería visualizar como aprendiz que domina las habilidades lingüísticas, lector moderno que sabe procesar textos y a través de ellos elige, dialoga, interpreta, confronta y comprende el conocimiento, lo incrementa y lo transforma; y como aprendiz que se forja continuamente buscando ser competente, crítico lector y escritor que re-aprende no solo a leer, sino a usar y dominar las estrategias de aprendizaje, lectura y escritura encaminadas a la comprensión, re-construcción y transformación de la información en conocimiento (Solé, 2012); lee para aprender y aprende a disfrutar de la lectura.

De igual forma, se hace oportuno el intercambio entre las redes, los grupos y las líneas de investigación interesadas en el aprendizaje o la enseñanza en educación superior; en lo relativo con la comprensión y aprendizaje del objeto de estudio disciplinar observable en la producción textual, las prácticas de lectura y escritura y su relación con el cambio conceptual, 
la comparación entre las prácticas de lectura y escritura de expertos y novatos-aprendices, entre otras; buscando teorías, diseños de evaluación o didácticas innovadoras, que apoyadas en la psicología, la lingüística, la didáctica y la pedagogía (diálogo interdisciplinar que oriente decisiones) valoren, fomenten, mantengan y fortalezcan los procesos, los resultados y las competencias de lectura y escritura de los estudiantes de educación superior.

En el "deber ser" nos compete a todos los que trabajamos en educación, aportar para mejorar las competencias de la lectura y la escritura integradas al proceso de aprendizaje, y de paso democratizar el conocimiento, porque una de las razones sociales de la educación es servir para que los sujetos conozcan, lean, critiquen, transformen y escriban sobre su realidad social, cultural e histórica.

Concluyendo, la lectura y la escritura se deben priorizar en la educación superior como prácticas académicas, requeridas de manera explicita y sistemática en la enseñanza y en la evaluación; fortalecidas a través de las estrategias cognitivas y meta-cognitivas, y la representación retórica de las tareas (recursos conceptuales y lingüísticos) en situaciones comunicativas complejas; en procura de beneficiar la objetivación y el distanciamiento del propio discurso, la revisión crítica de las ideas, la restructuración de los datos en conocimiento conceptual o procedimental y su reorganización para la discusión, la aplicación y la transferencia a nuevos contextos o escenarios, la formulación de hipótesis, la solución de problemas (personales, profesionales y laborales), es decir, la apropiación, reconstrucción y comunicación comprensiva del aprendizaje disciplinar. Cultura académica con liderazgo en los lineamientos de formación profesional de los estudiantes a maestro, encargados de favorecer las condiciones para que sus aprendices, comprendan y usen los procedimientos que subyacen a dichas prácticas, beneficiando la comprensión significativa de conocimientos.

\section{Referencias bibliográficas}

Aterrosi, A. (Dir.) (2009). Aportes para la enseñanza de la lectura. Segundo estudio regional comparativo y explicativo. Oficina Regional de Educación para América Latina y el Caribe (OREALC/UNESCO Santiago), y Laboratorio de Evaluación de la Calidad de la Educación (LLECE). Chile: Salesianos impresores. Recuperado de http://portal.unesco.org/ geography/es/ev.php-URL_ID=10936\&URL_ $\mathrm{DO}=\mathrm{DO} \_T O P I C \& U R L \_S E C T I O N=201$. html [Consultado el 15 de mayo de 2012].

Aterrosi, A. (Dir.) (2010). Escritura. Un estudio de las habilidades de los estudiantes de América Latina y el Caribe. Oficina Regional de Educación para América Latina y el Caribe (OREALC/UNESCO, Santiago), Laboratorio de Evaluación de la Calidad de la Educación (LLECE). Chile: Productora Gráfica Andros Ltda. Recuperado de http://portal.unesco.org/ geography/es/ev.php-URL_ID=14284\&URL_ DO=DO_TOPIC\&URL_SECTION=201. html [Consultado el 15 de mayo de 2012]

Cano, F. (2005). Consonance and dissonance in students' learning experience, p.p. 201-223. En Learning and Instruction 15. Recuperado de www.elsevier.com/locate/learninstruc [Consultado el 16 de mayo de 2012].

Cardona, G. (1994). Antropología de la escritura. Barcelona: Gedisa.

Carlino, P. (2004, julio-agosto). El proceso de escritura académica: cuatro dificultades de la enseñanza universitaria. Educere, 8 (026), 321- 
327. Red de Revistas Científicas de América Latina y el Caribe. Venezuela: Universidad de los Andes, recuperado de http://redalyc.uaemex.mx/ [Consultado el 2 de agosto de 2012].

Carlino, P. (2005a). Representaciones sobre la escritura y formas de enseñarla en universidades de América del Norte. Revista de Educación, (336), 143-168.

Carlino, P. (2005b). Escribir, leer, y aprender en la universidad. Una introducción a la alfabetización académica. Buenos Aires: Fondo de Cultura Económica.

De Zubiría Samper, M. (1997). Teoría de las seis lecturas. Mecanismos de aprendizaje semántico. Tomo I: preescolar y primaria. Bogotá: Fondo de publicaciones Bernardo Herrera Merino.

De Zubiría Samper, M. (1998). Teoría de las seis lecturas. Mecanismos de aprendizaje semántico. Cómo enseñar a leer y escribir ensayos. Tomo II: Bachillerato y Universidad. Bogotá: Fondo de publicaciones Bernardo Herrera Merino.

Flower, L. and Hayes, J. (1980). The dynamics of composing: Making plans and juggling constraints. En L. Gregg y E. Steinberg (Eds.). Cognitive processes in writing (pp. 31-50). Hillsdale, New Jersey: L. Erlbaum.

Gutiérrez Ríos, Y. (2012, mayo-agosto). Ausencia de una enseñanza reflexiva y sistemática de la oralidad. Revista Iberoamericana de Educación, 59, 223-239. Recuperado de http://www.rieoei. org/rie59a11.pdf [Consultado el 12 de agosto de 2012].

Kozulin, A. (2000). Instrumentos psicológicos. Barcelona: Paidós.
Martínez Fernández, J. R. (2004). Concepción de aprendizaje, metacognición y cambio conceptual en estudiantes universitarios de psicología. Tesis Doctoral. Universidad de Barcelona, Facultat de Psicología, Departament de Psicología Básica.

Martínez, J.R., y Galán F. (2000, primer semestre). Estrategias de aprendizaje, motivación y rendimiento académico. REOP, 11 (19), 3550.

Míguez, M. (2005, julio-diciembre). El núcleo de una estrategia didáctica universitaria: motivación y compresión. Revista ieRed, 1 (3). Revista Electrónica de la Red de Investigación Educativa [en línea]. Recuperado de http://revista.iered. org [Consultado el 15 de septiembre de 2011]. Ochoa, S. y Aragón, L. (2004). Funcionamiento meta-cognitivo de estudiantes universitarios durante la lectura de artículos científicos. Recuperado de http://pepsic.bvsalud.org/pdf/ up/v6n3/v6n3a03.pdf [Consultado el 15 de octubre de 2011].

Pozo, J. I.; Scheuer, N. y Mateos, M. (Comps.) (2009). La definición curricular del sistema nacional de Educación Superior Tecnológica. En Taller de instrumentación didáctica para la formación y el desarrollo de competencias profesionales (pp. 6-40).

Riestra, D. (2004). Las consignas de trabajo en el espacio socio-discursivo de la enseńanza de la lengua. [Tesis de Doctorado]. Genève, Université de Genève.

Rincón, G., Narváez, E. y Pérez, M. (2011). Construyendo un proyecto de investigación sobre la lectura y la escritura, mientras leemos y escribimos. Investigación en proceso: ¿Para qué se lee y se escribe en la universidad colombiana? Un aporte a la consolidación de la cultura académica en el país. Colciencias, y 17 Universidades Colombianas. Recuperado 
Tdea Patricia Ramírez. Otálvaro

de http://www.docstoc.com/docs/42204222/ CONSTRUYENDO-UN-PROYECTO-DEINVESTIGACION-SOBRE-LECTURA-YESCRITURA\# [Consultado el 1 de agosto de 2012]

Sánchez Lobato, Jesús (Coord.). (2007). Saber escribir. Colombia: Santillana USA. Instituto Cervantes.

Solé, I., y Coll, C. (2006). Los profesores y la concepción constructivista. En Coll, C., Martín, T., Mauri, M., Onrubia, J., Solé, I. y Zabala, A. El constructivismo en el aula, (pp. 7-23). 16 ed. Barcelona: Graó.

Solé, I. (2012, mayo-agosto). Competencia lectora y aprendizaje. Revista Iberoamericana de Educación, 59, 43-62. Recuperado de http:// www.rieoei.org/rie59a02.pdf [Consultado el 12 de agosto de 2012].
Ugartetxea, J. (2001). Motivación y metacognición, más que una relación. RELIEVE, V., 7 (2), 51-71. Recuperado de http://www. uv.es/RELIEVE/v7n2/RELIEVEv7n2_1.htm [Consultado el 03 de febrero de 2012].

Valery, O. (2000, junio). Reflexiones de la escritura a partir de Vigotsky. Educere, 3 (9). Recuperado de http://redalyc.uaemex.mx/ pdf/356/35630908.pdf [Consultado el 13 de febrero de 2012].

Van Dijk, T.A. (1985). La ciencia del texto. Barcelona: Paidós.

Zambrano, J. (2010). Los docentes y el proceso de escribir antes y durante los estudios universitarios. Universidad de Táchira. Recuperado de http:// saber.ula.ve [Consultado el 15 de febrero de 2012]. 